\title{
Single Incision Laparoscopic Surgery in Pregnancy
}

\author{
Ismail A. Al-Badawi, MD, Hanan Alshankiti, MD, Sarfraz Ahmad, PhD \\ King Faisal Specialist Hospital and Research Center, Department of Obstetrics and Gynecology, Riyadh, Saudi Arabia \\ (Drs Al-Badawi and Alshankiti). \\ Florida Hospital Cancer Institute, Department of Gynecologic Oncology, Orlando, FL, USA (Dr Ahmad).
}

\begin{abstract}
Laparoendoscopic single-site surgery, also known as single incision laparoscopic surgery (SILS), is advancing the minimally invasive surgical approach. Herein, we describe our experience with 2 cases using the SILS in pregnancy and discussing the relevant peer-reviewed English literature. Case 1: A 42-year-old woman, who was pregnant at 17-weeks gestational age, presented with sudden onset right lower abdominal pain. Pelvic ultrasonogram characteristic revealed right large adnexal cyst measuring $12 \mathrm{~cm} \times 12 \mathrm{~cm} \times 7 \mathrm{~cm}$ with torsion. Diagnosis of ovarian cyst with torsion was made, which indicated surgical intervention during pregnancy. SILS was performed using a single port through a 2-cm umbilical incision to the peritoneal cavity. Right ovarian cyst with torsion was identified. Untwisting of the torted pedicle and ovarian cystectomy was performed, the patient had a spontaneous vaginal delivery at 38 weeks. Case 2: A 27-year-old woman, 12 weeks pregnant, presented through the emergency department with severe pain, and a pelvic ultrasonogram showed a 10-cm left adnexal mass with suspicion of torsion. Emergency diagnostic laparoscopy was performed using SILS with the finding of torted left ovarian cyst. Ovarian cystectomy and untwisting of the adnexa was performed, the patient had a preterm delivery of a stillborn at 20 weeks plus 4 days. We conclude with our successful experiences of SILS in pregnancy without any complications. This report is among the early experiences using SILS in pregnancy and is likely be the first from the Arab world (Middle East) experience. Further studies would be desirable to determine short-term and long-term outcomes.
\end{abstract}

Key Words: Case reports, Gynecologic surgery, Laparoscopy, Pregnancy, Single incision.

Citation Al-Badawi IA, Alshankiti H, Ahmad S. Single incision laparoscopic surgery in pregnancy. CRSLS e2014.00404. DOI: 10.4293/CRSLS.2014.00404.

Copyright (c) 2014 SLS This is an open-access article distributed under the terms of the Creative Commons Attribution-Noncommercial-ShareAlike 3.0 Unported

license, which permits unrestricted noncommercial use, distribution, and reproduction in any medium, provided the original author and source are credited.

Address correspondence to: Ismail A. Al-Badawi, MD, Department of Obstetrics and Gynecology, King Faisal Specialist Hospital and Research Center, PO Box 3354,

Riyadh 11211, MBC-52, Saudi Arabia. Telephone: +966-1-442-4885, Fax: +966-1-442-7393, E-mail: i_albadawi@yahoo.com

\section{INTRODUCTION}

Over the years, there have been many advances in the field of minimally invasive surgery. Particularly, laparoendoscopic single-site surgery (LESS), which is also referred to as single incision laparoscopic surgery (SILS), describes the use of 1 small skin incision to complete laparoscopic surgical procedures in which, traditionally, multiple incisions are created.

Although, SILS was first described over 2 decades ago, but gynecologists have been slower in adopting the SILS approach for wider applications/indications. ${ }^{1}$ SILS is a promising technique that is feasible and relatively safer when performed by experienced laparoscopic surgeons and can be offered for a variety of gynecologic procedures, including for the treatment of select malignancies. ${ }^{2}$

Recent comparison series and 2 randomized controlled trials suggested some improved cosmetic results, reduced postoperative pain, and analgesia when SILS was compared with the traditional laparoscopic approach. ${ }^{3-7} \mathrm{How}^{-}$ ever, in the peer-reviewed English literature, there is a limited reported experience where the SILS (LESS) approach has been used in pregnancy. Kim and Kwon ${ }^{8}$ reported a similar case using LESS to describe a surgical approach for the management of a pregnant woman with an ovarian tumor using cyst exteriorization outside the umbilical incision. More recently, Dursum et al ${ }^{9}$ reported the feasibility of the LESS approach for the management of huge adnexal cysts and adnexal torsion during pregnancy in 2 patients. These early observations concluded that LESS in pregnant patients with adnexal mass is feasible and might be a better surgical option for the pregnant patients with adnexal pathology. 8,9

Herein, we report our successful experience of 2 cases using SILS in pregnancy without any complications, 
which highlights the possibility of considering this approach for future interventions in gynecological surgeries during pregnancy. This is among the early experiences of SILS in pregnancy and is likely be the first report from the Arab world (Middle East) in the peerreviewed literature.

\section{CASE DESCRIPTION/TECHNIQUE DESCRIPTION}

\section{Case 1}

A 42-year-old woman, who was pregnant at 17-weeks gestational age, presented with sudden onset right lower abdominal pain at our primary clinic. She had no nausea, vomiting, anorexia, or vaginal bleeding. All her previous pregnancies were normal vaginal deliveries without any reported complications. Her past medical and surgical history was unremarkable.

On examination, she was found to be febrile and tachycardic. Her body mass index was $40 \mathrm{~kg} / \mathrm{m}^{2}$, with abdominal tenderness over the lower abdomen (mainly right side), and no rebound tenderness. Her lab results were: white blood count 13,000, and hemoglobin 106 g/L. Pelvic ultrasonogram characteristic revealed right large adnexal cyst measuring $12 \mathrm{~cm} \times 12 \mathrm{~cm} \times 7 \mathrm{~cm}$ with torsion. Therefore, the diagnosis of ovarian cyst with torsion was made, which indicated surgical intervention during the pregnancy.

Options of laparotomy versus laparoscopy were discussed with the patient (and also with the appropriate family members) at length, including the potential risks such as abortion, injury of the gravid uterus, postoperative pain, and prolonged hospitalization. The option of SILS approach was also discussed with her due to the cyst site and the limited space available for using the conventional laparoscopy; hence, there was no space for multiple ports. She eventually agreed on the SILS option, which was performed on an emergency basis.

A single port was introduced through a 2-cm umbilical incision to the peritoneal cavity (Figure 1). A 2-cm vertical incision was made in the umbilicus at the beginning of the operation. Layer by layer, the peritoneal cavity was entered; then 0 polyglactin 910 (Vicryl; Ethicon, Inc, Somerville, New Jersey) sutures were placed at each side of the fascia (as stay sutures to help in final closure at the end of the procedure). The SILS device was inserted into the wound opening transumbilically. After insertion of the SILS device into the abdominal cavity, 35-mm sheaths were inserted through the device. Carbon dioxide gas was insufflated through the special connector to the device (Figure 1B).

Intra-abdominal pressure was maintained at $12 \mathrm{~mm} \mathrm{Hg}$. Once pneumoperitoneum was achieved using $2.5 \mathrm{~L}$ of carbon dioxide, no leakage of carbon dioxide from the connected portions was noted. Intra-abdominal visualization was obtained with a $5-\mathrm{mm} 0^{\circ}$ laparoscope (Pano-view; Richard Wolf GmbH, Knittlingen, Germany) inserted through a $5-\mathrm{mm}$ cannula. No intrauterine instruments were used. Carefully and slowly, the patient was placed in the Trendelenburg position. At the abdominal entry, initial inspection revealed no adhesions, a gravid uterus 17 gestational weeks in size, a normal left adnexa, and the whitish glistening smooth surface of a torted right ovarian tumor. The tumor was grasped using conventional laparoscopic atraumatic grasping forceps and untorsion of the cyst was achieved. Then, the ovarian mass was pulled toward the umbilical incision. The ovarian cyst was punctured with a curved Metzenbaum scissors, and its contents were aspirated using a suction irrigation apparatus. To prevent cyst spillage, the puncture site on the cyst was held and pushed up against the abdominal wall with a forceps. Then, the deflated cyst was exteriorized through the umbilical wound, and at the same time, the SILS device was removed from the umbilical wound. After the ovarian tumor was removed, carbon dioxide insufflations were stopped. A right ovarian cystectomy was then performed using the traditional technique outside the abdominal cavity, with preservation of as much ovarian tissue as possible (Figure 2C).

The right ovary was placed back in the peritoneal cavity; the SILS device was reattached to the umbilical wound; and pneumoperitoneum was re-established. Irrigation and hemostasis were then performed under laparoscopic control. The peritoneum and fascia were closed layer by layer with the 0 polyglactin 910 (Vicryl) sutures, and the skin was closed with 4-0 polyglactin 910 sutures. The fetus tolerated the operative intervention well, with a fetal heartbeat of about 150 beats/minute (both before and just after the operation).

Throughout the procedure, we did not encounter any bleeding or intraoperative complications. The operative time was 40 minutes with minimal blood loss $(<100 \mathrm{~mL})$. Notably, intensive care unit service was not required, and the patient was discharged for home on postoperative day 2 with positive fetal heart, no signs of uterine contraction, minimal pain, and on a regular diet. No tocolytic agent 
A.

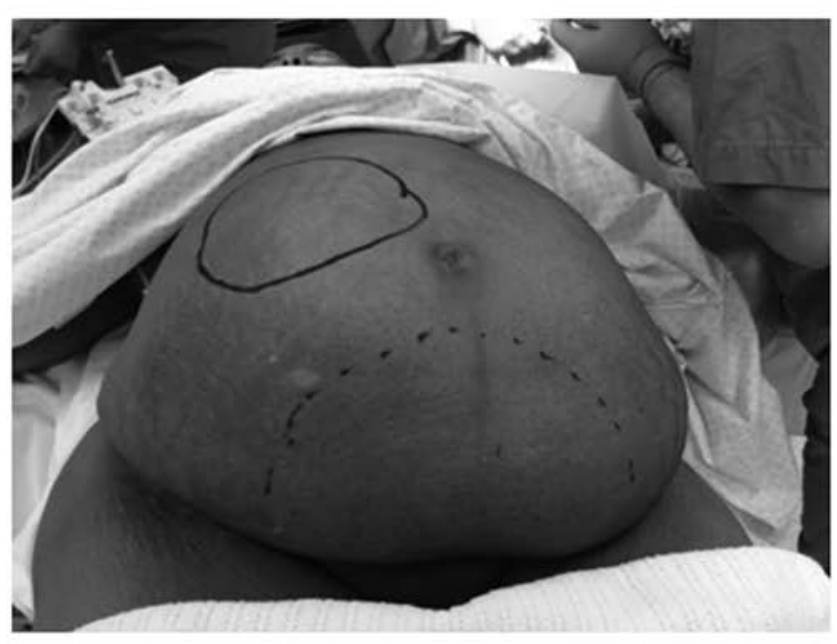

\section{C.}

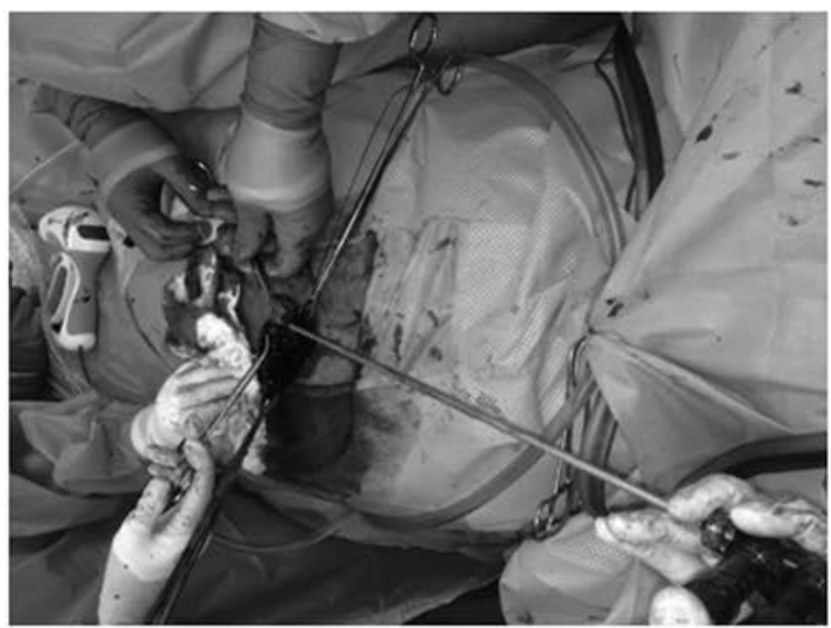

B.

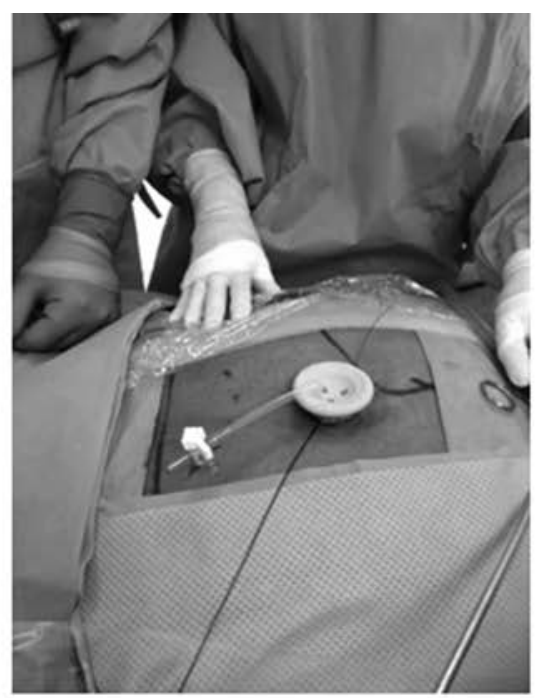

D.

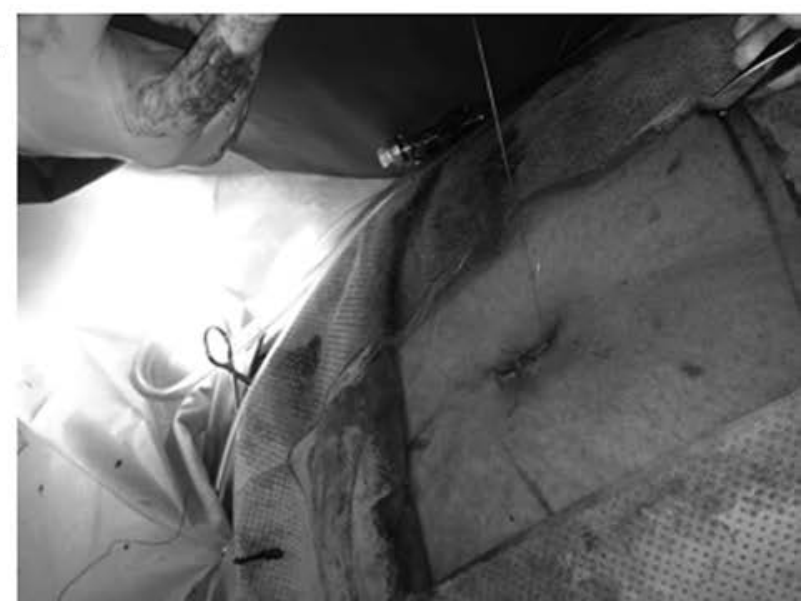

Figure 1. A. The site of the twisted ovarian mass at right upper quadrant and the dotted line represent the pregnant uterus location. B. View of the SILS device insertion. C. Ovarian cystectomy using exteriorization method. D. View of closing the skin incision.

was required, and the postoperative surgical wounds demonstrated satisfactory cosmetic results. Final pathology came as benign serous cyst adenoma with evidence of torsion. The patient had a spontaneous vaginal delivery of a healthy baby boy at 38 weeks.

\section{Case 2}

A 27-year-old lady, at 12 weeks of gestation, presented to the emergency department with a complaint of severe lower abdominal pain. She was admitted at a peripheral hospital where she was under observation and given analgesics without any other interventions. She was not getting better, so she signed herself out against the medical advice. We admitted the patient, and routine blood work and pelvic ultrasonogram were done. Her hemoglo- bin level was $81 \mathrm{~g} / \mathrm{L}$ (it was $100 \mathrm{~g} / \mathrm{L}$ at the referring hospital), and potassium level was $2.8 \mathrm{mmol} / \mathrm{L}$. She was transfused with $2 \mathrm{U}$ of packed red blood cells, and her potassium was corrected. The ultrasonogram findings were consistent with a viable intrauterine pregnancy corresponding to 12 weeks of gestation and a $10-\mathrm{cm}$ adnexal mass posterior to the uterus pressing on the rectum.

The peripheral vascularity was preserved, but there it was not clear inside the mass. A magnetic resonance imaging scan was suggested, but the recommendation was not to use dye for the study because of the pregnancy. The treating team felt it would not be useful to do the magnetic resonance imaging scan without the dye, and the patient was in severe pain requiring fentanyl patient-controlled analgesia, so following the appropriate consents, she was 
A.

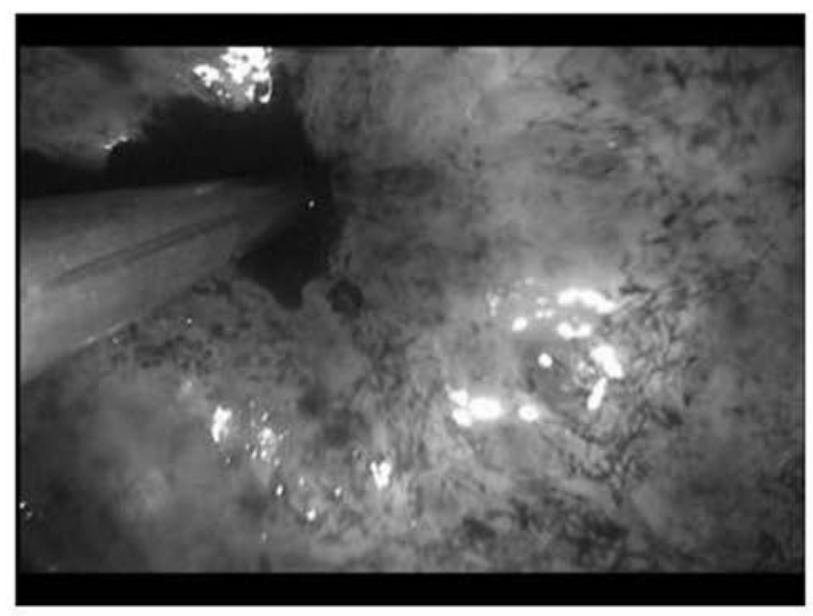

c.

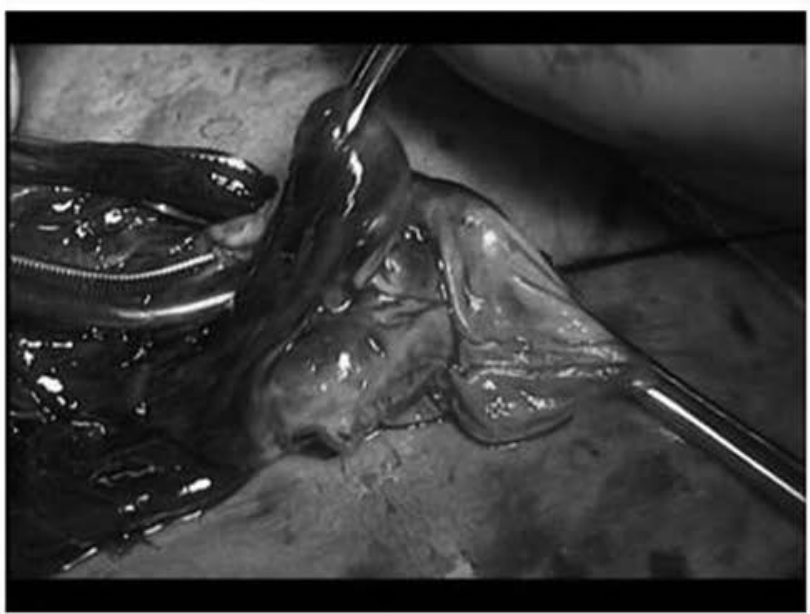

B.

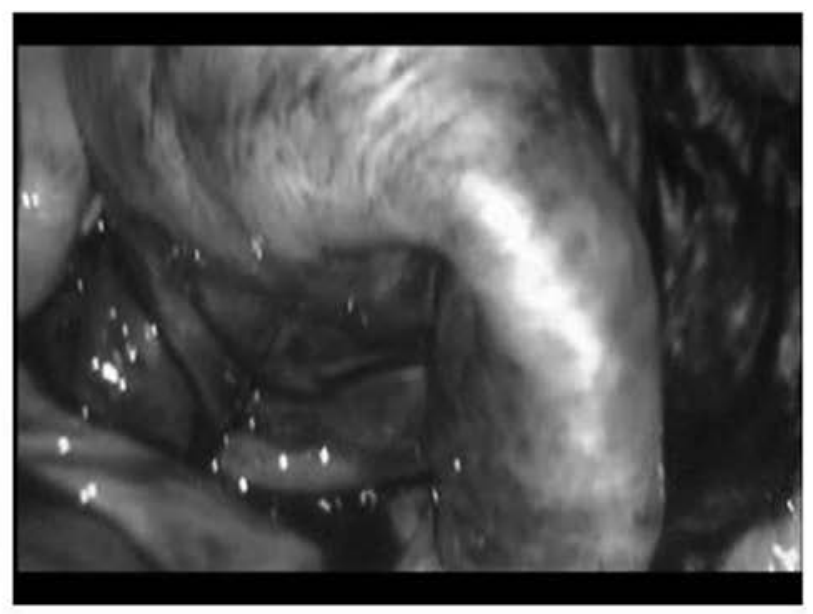

D.

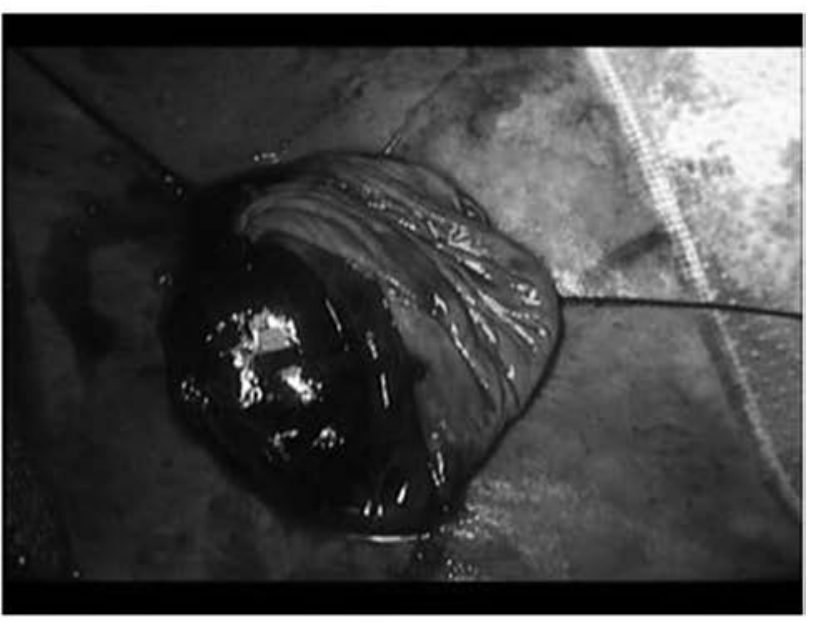

Figure 2. A. View from the aspiration of the cyst fluid. B. View of the ovarian infundibulopelvic ligament torsion. C. View of the ovarian cystectomy through the SILS skin incision. D. View of the ovarian cystectomy completion.

taken for an emergency SILS for ovarian cystectomy, and possible oophorectomy.

A 2-cm umbilical incision was made and the SILS device was introduced. The carbon dioxide pressure was set at $12 \mathrm{~mm} \mathrm{Hg}$. Using a 5-mm, $0^{\circ}$ scope and 2 laparoscopic graspers, the cyst was untwisted and ovarian cystectomy was performed using a 5-mm ligature (vessel sealing device). The color of the ovary had improved from dark bluish into pinkish color toward the end of the procedure. Blood loss was minimal $(<100 \mathrm{~mL})$, and the operative time was only 40 minutes.

The pain had improved dramatically postoperatively; the umbilical incision was clean and dry. The final pathology came back as simple cyst with evidence of hemorrhage and torsion. The patient had a preterm delivery of a stillborn at 20 weeks plus 4 days.

\section{DISCUSSION}

In this report, we described our successful experience of SILS in pregnancy without any complications, which highlights the possibility of considering the SILS option for interventions in gynecological surgeries during pregnancy. The SILS approach was not only feasible in these 2 pregnant women, but it also turned out to be a much better choice because the first patient was morbidly obese with pregnancy and twisted ovarian mass, which was located in the right upper abdomen because of the pregnancy effect. The surgical options were either to do a large incision laparotomy with its morbidity or to perform panniculectomy for the patient's large pannus at the time of laparotomy. Alternatively, we could have opted to perform a conventional multiport laparoscopy, which would have resulted in a very limited access inside the abdomen 
and we might not have been able to use the skin incision of the SILS device to complete the cystectomy and remove the specimen. We performed 2 cases of SILS for the management of ovarian torsion with cystectomies in pregnant women by using an exteriorization method. The 10-cm cyst was accessed through a SILS device; grasped; delivered through the umbilical incision, where cystectomy was performed; and placed back into the peritoneal cavity.

There have been reports in which similar extracorporeal methods were used in the management of adnexal masses. ${ }^{8}$ We believe this method could avert inadvertent injuries to the enlarged uterus in pregnant women from the initial insertion of the insufflation needle or trocar. We also believe that the exteriorization method of transumbilical management is feasible in pregnant women for several reasons. For example, in pregnancy, as the uterus enlarges, the ovaries situate outside the pelvis and are much closer to the umbilicus. Usually, because the adnexal ligaments are overstretched during pregnancy, ${ }^{10}$ it is possible to move the adnexa to the periumbilical area, where the enlarged uterus is less troublesome. Furthermore, ovarian tumors can be more effectively removed in the larger umbilical wound incision in SILS than they can be in the $0.5-\mathrm{cm}$ or $1.2-\mathrm{cm}$ ports used in the traditional laparoscopy procedure. ${ }^{11}$ Thus, we believe that our approach of using the SILS with exteriorization is easier and more suitable in pregnant women than the conventional laparoscopic methods.

Surgery in pregnancy carries added risks, including the fetal loss, preterm contractions, and/or an increased risk of embolic events. Furthermore, the most appropriate/ traditional surgical routes (ie, laparotomy or laparoscopy) have not been defined, and thus in many cases observation can be warranted. While the main indication for intervention in pregnancy is adnexaltorsion, ${ }^{12}$ the most common indications of laparoscopy in pregnancy are cholelithiasis, appendicitis, persistent ovarian cyst, and adnexal torsion. ${ }^{13}$

The peer-reviewed literature supporting the surgical management suggests a trend toward the improved fetal and maternal outcomes by using mid-gestation (12-27 weeks) laparoscopy when operating on patients with adnexal masses that are suspicious for malignancy or when clinically symptomatic. ${ }^{14,15}$ Investigators in favor of a laparotomy approach raise several concerns regarding the laparoscopic approach in pregnancy, including the lack of data regarding the effects of a pneumoperitoneum; possible injection of carbon dioxide into the uterine cavity; possible injury to the gravid uterus by a Veress needle, trocar, or surgical instrument; and the potential for fetal acidosis because of maternal conversion of carbon dioxide to carbonic acid.

On the other hand, advocates of laparoscopy emphasize decreased postoperative pain, less narcotic use, shorter hospital length-of-stay, and less need for uterine traction, leading to less uterine irritability associated with laparoscopy. Furthermore, laparoscopy results in faster postoperative ambulation and return to regular activity, which are very important in pregnancy because of the potential for increased rate of thromboembolic events. ${ }^{10}$ However, multiple observational studies have demonstrated that laparoscopic management of adnexal masses in pregnancy is technically feasible and should no longer be considered contraindicated in pregnancy. ${ }^{11,14-17}$

The Society of American Gastrointestinal and Endoscopic Surgeons published some recommendations that are specific to performing laparoscopic procedure during pregnancy, including intraoperative carbon dioxide monitoring by capnography. Although, use of the Veress needle is not contraindicated, the surgeon may consider using the Veress needle in conjunction with ultrasonogram guidance, which is not the case with SILS technique. Intraoperative abdominal pressure should be maintained at $<15$ $\mathrm{mm} \mathrm{Hg}$ while in the Trendelenburg position to ensure adequate venous return and uteroplacental sufficiency. ${ }^{10}$

Recent research has suggested potential advantages of SILS over conventional multiport laparoscopy. ${ }^{18}$ These advantages include better cosmetic results because of a relatively hidden umbilical scar and the need for fewer trocar incisions, a possible decrease in morbidity related to the visceral and vascular injuries during trocar placement, reduced risk of postoperative wound infections and hernia formation, and elimination of multiple trocar site closures. Another potential merit of SILS is the reduction of postoperative pain and narcotic use, which we believe is important in pregnant women.

In conclusion, the feasibility of SLIS approach seems encouraging as we report our successful experience of SILS in pregnancy without any complications. We achieved good cosmesis with minimal discomfort, and the patients are currently having a better quality-of-life with the pregnancy outcomes in the first patient, and the second patient is looking forward to getting pregnant after her pregnancy loss. Because this report supports the previous limited experiences using the SILS (LESS) in pregnancy, further prospective studies that compare traditional laparoscopy with SILS would be desirable to determine short-term and long-term clinical outcomes. 


\section{References:}

1. Pelosi MA, Pelosi MA 3rd. Laparoscopic supracervical hysterectomy using a single-umbilical puncture (mini-laparoscopy). J Reprod Med. 1992;37(9):777-784.

2. Fader AN, Rojas-Espaillat L, Ibeanu O, Grumbine FC, Escobar PF. Laparoendoscopic single-site surgery (LESS) in gynecology: a multi-institutional evaluation. Am J Obstet Gynecol. 2010; 203(5):501.e1-6.

3. Kim TJ, Lee YY, Cha HH, et al. Single-port-access laparoscopic-assisted vaginal hysterectomy versus conventional laparoscopic-assisted vaginal hysterectomy: a comparison of perioperative outcomes. Surg Endosc. 2010;24(9):2248-2252.

4. Yim GW, Jung YW, Paek J, et al. Transumbilical single-port access versus conventional total laparoscopic hysterectomy: surgical outcomes. Am J Obstet Gynecol. 2010;203(1):26e1-6.

5. Fagotti A, Rossitto C, Marocco FG, et al. Perioperative outcomes of laparoendoscopic single-site surgery (LESS) versus conventional laparoscopy for adnexal disease: a case-control study. Surg Innov. 2011;18(1):29-33.

6. Fagotti A, Bottoni C, Vizzielli G, et al. Postoperative pain after conventional laparoscopy and laparoendoscopic single site surgery (LESS) for benign adnexal disease: a randomized trial. Fertil Steril. 2011;96(1):255.e2-259.e2.

7. Chen YJ, Wang PH, Ocampo EJ, Twu NF, Yen MS, Chao KC. Single-port compared with conventional laparoscopic-assisted vaginal hysterectomy: a randomized controlled trial. Obstet Gynecol. 2011;117(4):906-912.

8. Kim WC, Kwon YS. Laparoendoscopic single-site surgery for exteriorization and cystectomy of an ovarian tumor during pregnancy. J Minim Invasive Gynecol. 2010;17(3):386-389.
9. Dursun P, Gülümser C, Cağlar M, Araz C, Zeyneloğlu H, Haberal A. Laparoendoscopic single-site surgery for acute adnexal pathology during pregnancy: preliminary experience. $J$ Matern Fetal Neonatal Med. 2013;26(13):1282-1296.

10. Soper NJ. SAGES' guidelines for diagnosis, treatment, and use of laparoscopy for surgical problems during pregnancy. Surg Endosc. 2011;25(11):3477-3478.

11. Moore RD, Smith WG. Laparoscopic management of adnexal masses in pregnant women. J Reprod Med. 1999;44(2):97-100.

12. Morice P, Louis-Sylvestre C, Chapron C, Dubuisson JB. Laparoscopy for adnexal torsion in pregnant women. J Reprod Med. 1997;42(7):435-439.

13. Al-Fozan H, Tulandi T. Safety and risks of laparoscopy in pregnancy. Curr Opin Obstet Gynecol. 2002;14(4):375-379.

14. Mathevet P, Nessah K, Dargent D, Mellier G. Laparoscopic management of adnexal masses in pregnancy: A case series. Eur J Obstet Gynecol Reprod Biol. 2003;108(2):217-222.

15. Yuen PM, Ng PS, Leung PL, Rogers MS. Outcome in laparoscopic management of persistent adnexal mass during the second trimester of pregnancy. Surg Endosc. 2004;18:1354-1357.

16. Lenglet $\mathrm{Y}$, Roman H, Robishong B, et al. Laparoscopic management of ovarian cysts in pregnancy [in French]. Gynecol Obstet Fertil. 2006;34(2):101-106.

17. Rizzo AG. Laparoscopic surgery in pregnancy: long-term follow-up. J Laparoendosc Adv Surg Tech A. 2003;13(1):11-15.

18. Kim TJ, Lee YY, Kim MJ, et al. Single-port access laparoscopic adnexal surgery. J Minim Invasive Gynecol. 2009;16(5):612-615. 